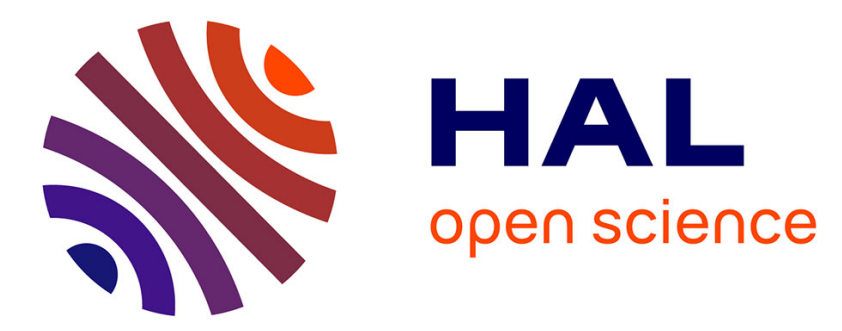

\title{
Remarques sur la théorie des propriétés magnétiques des substances dures
}

Louis Néel

\section{To cite this version:}

Louis Néel. Remarques sur la théorie des propriétés magnétiques des substances dures. Applied Scientific Research, Section B, 1955, 4 (1), pp.13-24. 10.1007/BF02919980 . hal-02888359

\section{HAL Id: hal-02888359 \\ https://hal.science/hal-02888359}

Submitted on 29 Jul 2020

HAL is a multi-disciplinary open access archive for the deposit and dissemination of scientific research documents, whether they are published or not. The documents may come from teaching and research institutions in France or abroad, or from public or private research centers.
L'archive ouverte pluridisciplinaire HAL, est destinée au dépôt et à la diffusion de documents scientifiques de niveau recherche, publiés ou non, émanant des établissements d'enseignement et de recherche français ou étrangers, des laboratoires publics ou privés. 


\title{
REMARQUES SUR LA THEORIE DES PROPRIETES MAGNETIQUES DES SUBSTANCES DURES
}

\author{
par LOUIS NÉEL
}

Faculté des Sciences, Institut Fourier, Place du Doyen Gosse, Grenoble, France

\section{Som maire}

L'auteur montre qu'il est possible d'améliorer d'une manière très simple la théorie de l'hystérésis magnétique des grains fins monodomaines en tenant compte, d'une part de la dispersion du champ coercitif des grains individuels, et d'autre part du champ d'interaction entre les grains et de la dispersion de ce champ autour de sa valeur moyenne. On réussit ainsi à rendre compte des caractéristiques principales du cycle d'hystérésis et à interpréter notamment les lois de Rayleigh relatives aux champs faibles: on obtient, sans aucune donnée arbitraire initiale, des valeurs numériques qui concordent d'une manière remarquable avec les données expérimentales relatives aux bons aimants permanents connus.

$\S 1$. La théorie des grains monodomaines. Les processus d'aimantation des substances ferromagnétiques se ramènent à deux mécanismes élémentaires: soit à des déplacements des parois de séparation entre domaines élémentaires de Weiss, soit à une rotation d'ensemble des moments magnétiques de ces domaines. C'est ce dernier mécanisme qui joue sans doute un rôle prépondérant lorsqu'il s'agit de substances magnétiques dures, telles celles qui constituent les bons aimants permanents actuels. Les phénomènes sont particulièrement simples à interpréter lorsque la substance contient deux phases dont l'une, non magnétique, constitue une matrice qui enrobe les grains magnétiques de la seconde phase. Lorsque ces grains sont suffisamment petits, c'est-à-dire de l'ordre du dixième de micron ou moins, ils ne contiennent qu'un seul domaine élémentaire de Weiss: le moment magnétique résultant du grain conserve donc une valeur constante, seule son orientation peut changer. C'est ainsi que dans les aimants en poudre de fer comprimée, la phase magnétique est constituée par les grains de fer tandis que la matrice est formée par les 
cavités qui subsistent entre les grains après la compression. De même, dans les aimants du type Fe-Ni-Al, on aurait affaire à une phase magnétique; de propriétés voisines de celles du fer, immergée dans une phase non magnétique formée principalement de nickel et d'alumium.

Les lois de l'aimantation d'un grain isolé morfodomaine sont bien connues $\left.\left.\left.{ }^{1}\right)^{2}\right)^{3}\right)^{4}$ ). En général, on suppose pour simplifier le grain de révolution de sorte que l'énergie totale du grain dans un champ $\mathbf{H}$ s'exprime, en désignant par $\theta$ l'angle du moment $\mathbf{M}$ du grain avec l'axe de révolution, par la formule

$$
W=-\mathbf{M H}-M H_{c} \cos ^{2} \theta,
$$

où $H_{c}$ est une constante, appelée champ critique, qui caractérise l'anisotropie magnétique du grain: anisotropie de forme (allongement du grain), anisotropie magnétocristalline (couplage du moment magnétique avec le réseau cristallin) ou encore anisotropie magnétoélastique (tensions internes). Il suffit d'écrire que $W$ est minimum.

Lorsque le champ appliqué est parallèle à l'axe du grain, l'aimantation est une fonction du champ magnétique représentable par un cycle d'hystérésis rectangulaire, symétrique par rapport à l'origine et caractérisée par deux discontinuités d'aimantation correspondant aux champs $-H_{c}$ et $+H_{c}$. Lorsque le champ appliqué est perpendiculaire à l'axe, la composante $m$ du moment suivant la direction de $H$ varie réversiblement avec $H$ dans l'intervalle $-H_{c}<H<+H_{c}$ :

$$
m=\frac{M H}{H_{c}} .
$$

En dehors de cet intervalle, $m$ reste égal à $-M$ ou à $+M$ suivant que $H$ est plus petit que $-H_{c}$ ou plus grand que $+H_{c}$. Enfin, dans les cas intermédiaires où l'axe du grain est oblique par rapport à la direction du champ, on obtient un cycle d'hystérésis de forme complexe avec un champ coercitif et des discontinuités d'aimantation d'autant plus petits que l'axe est plus près d'être perpendiculaire au champ.

Si nous considérons maintenant un ensemble de grains identiques indépendants les uns des autres, avec des axes orientés au hasard, le cycle d'hystérésis moyen de l'ensemble est représenté par la courbe A de la figure 1 . Il est caractérisé par un moment magnétique rémanent égal à la moitié du moment à saturation et un champ coercitif 
égal à $0,48 H_{c}$, soit sensiblement la moitié du champ coercitif maximum: celui des grains d'axe parallèle au champ. Cette courbe présente évidemment quelques points de ressemblance avec les cycles d'hystérésis des substances dures: elle donne en particulier une valeur convenable pour l'aimantation rémanente ainsi que le bon ordre de grandeur du champ coercitif.

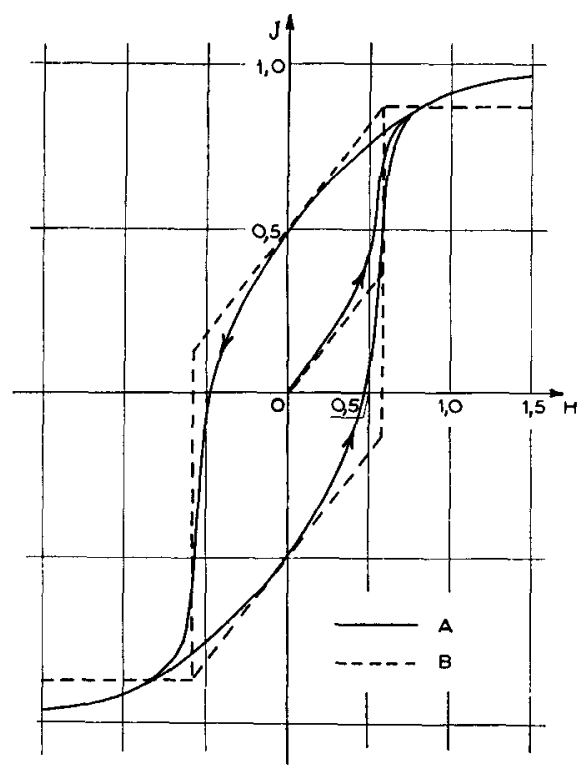

Fig. 1.

On pourrait d'ailleurs, sans trop compliquer, perfectionner la théorie en introduisant un ensemble de grains d'allongements divers convenablement répartis, depuis la sphère jusqu'au cylindre. Mais cela ne suffit pas à pallier les faiblesses de la théorie. Il est bien certain que pratiquement les grains ferromagnétiques sont beaucoup trop rapprochés les uns des autres pour être supposés indépendants. Malgré les complications qui vont en résulter, il faut donc abandonner l'hypothèse de l'indépendance des grains et rechercher dans quelle mesure l'introduction d'interactions modifie les résultats de la théorie simple et permet d'interpréter certains résultats expérimentaux qui sont incompatibles avec cette dernière.

Parmi ces incompatibilités, citons les lois de Rayleigh relatives aux champs faibles. En effet; d'après la théorie simple, si, après désaimantation, le champ magnétique ayant été amené à la valeur 
$H_{m}$, on ramène le champ de $H_{m}$ à 0 , l'aimantation devrait rester indépendante du champ tandis que l'expérience donne une variation parabolique. Remarquons également que le modèle simple possède une susceptibilité anhystérétique infinie tandis que l'expérience montre qu'elle est finie.

L'e f f e t d e proximit é. Il est possible d'évaluer l'influence des interactions sur le champ coercitif lorsque l'anisotropie des grains est simplement une anisotropie de forme. En effet, pour des grains isolés, de forme quelconque, orientés au hasard par rapport à la direction de l'aimantation spontanée $J_{0}$, l'énergie magnétostastique rapportée à $1 \mathrm{~cm}^{3}$ de grains, est égale à $\frac{2}{3} \pi J_{0}^{2}$. On montre d'autre part, étant donné n'importe quel système de cavités ou de grains de forme quelconque, soumis à la seule condition d'être en moyenne isotrope et aimanté à saturation, que son énergie magnétostatique est égale à $\frac{2}{3} \pi J_{0}^{2} v(1-v)$, en désignant par $v$ la fraction du volume total occupée par la phase magnétique. L'énergie, rapportée à l'unité de volume de cette phase vaut donc $\frac{2}{3} \pi J_{0}^{2}(1-v)$ : par suite de la proximité des voisins, l'énergie est donc multipliée par $(1-v)$. Le champ coercitif, proportionnel à l'énergie, est ainsi multiplié lui aussi $\operatorname{par}(1-v)$.

On prévoit de cette manière que le champ coercitif des aimants en poudre de fer comprimée doit être proportionnel à la densité moyenne de l'aggloméré: cette conclusion a été vérifiée d'une manière très satisfaisante par $\mathrm{W}$ e i $1^{5}$ ). La meilleure qualité de l'aimant correspond au maximum de l'énergie moyenne, c'est-à-dire au maximum de $v(1-v)$, soit $v=\frac{1}{2}$ : l'expérience montre qu'il en est bien ainsi.

$\mathrm{L}$ e $\mathrm{cham}$ p de Lor e $\mathrm{t} z$. L'effet de proximité ne constitue qu'un des multiples aspects du rôle des interactions entre les grains. Dans un ensemble de grains d'aimantation moyenne $J_{m}$, chaque grain est soumis, sous l'influence des grains voisins, à une sorte de champ de Lorentz d'intensité moyenne égale à $\frac{4}{3} \pi J_{m}$. Comme ce champ est nul au champ coercitif, il n'en modifie pas la valeur mais il déforme profondément les autres régions du cycle d'hystérésis: il correspond en effet, dans les substances qui nous intéressent, à des champs magnétiques considérables, voisins de 1800 u.é.m. au voisinage de l'aimantation rémanente.

Les fluctuations du champ de Lorentz. D'un grain à l'autre, ce champ subit d'importantes fluctuations de part et d'autre de la moyenne qu'il est possible d'évaluer en supposant qu'il 
n'existe aucune corrélation entre la direction des moments magnétiques de deux grains voisins et en supposant les grains répartis aux noeuds d'un réseau cubique à faces centrées de maille $a$. Le moment magnétique $m$ d'un grain est alors égal à $a^{3} J_{c} / 4$, en désignant par $J_{c}$ l'aimantation à saturation moyenne du système. Pour simplifier encore, supposons que le moment magnétique de chaque grain ne puisse occuper que deux orientations parallèle ou antiparallèle à la direction de l'intensité moyenne $J_{m}$. Si nous posons $p=J_{m} / J_{s}$, les probabilités respectives de ces deux orientations sont alors égales à $(1+p) / 2$ et $(1-p) / 2$.

Si nous considérons un domaine $\mathrm{B}$ tel que la ligne joignant le centre de $\mathrm{B}$ au centre d'un domaine voisin $\mathrm{A}$ fasse un angle $\theta$ avec la direction de l'aimantation résultante moyenne $J_{m}$, la composante moyenne $h_{m}$, suivant la direction de $J_{m}$, du champ $h$ créé par B en A est

$$
h_{m}=\frac{m}{r^{3}}\left(3 \cos ^{2} \theta-1\right) p \text { avec } r=\frac{a}{\sqrt{2}} .
$$

Le carré moyen de l'écart de $h$ par rapport à la moyenne $h_{m}$ est alors

$$
\overline{\left(h-h_{m}\right)^{2}}=\frac{m^{2}}{r^{6}}\left(3 \cos ^{2} \theta-1\right)^{2}\left(1-p^{2}\right),
$$

d'où finalement le carré moyen $H_{f}^{2}$ de l'écart du champ d'interaction par rapport à la moyenne du champ créé par les 12 voisins du domaine $\mathrm{A}$ :

$$
H_{j}^{2}=\frac{24}{5}\left(J_{s}^{2}-J_{m}^{2}\right)
$$

Un raisonnement analogue permet de déterminer le carré moyen $H_{i}^{\prime 2}$ de la composante du champ de fluctuation suivant une direction perpendiculaire à $J_{m}$; on trouve

$$
H_{f}^{\prime 2}=\frac{18}{5}\left(J_{s}^{2}-J_{m}^{2}\right)
$$

Ces deux dernières formules montrent que les fluctuations du champ de Lorentz sont les plus grandes au voisinage des états d'aimantation moyenne nulle $\left(J_{m}=0\right)$. L'amplitude de ces fluctuations, pour $J_{s}=850$, est alors de 1700 u.é.m. ; c'est une valeur considérable, de l'ordre de grandeur du champ critique des grains les plus 
anisotropes. Ces fluctuations doivent donc jouer un rôle important.

La probabilité de trouver le champ de fluctuation compris entre certaines limites est donnée par une loi de Gauss. Cependant, étant donnée la nature des approximations que nous serons amenés à faire plus loin, nous remplacerons pour simplifier cette loi de distribution par une loi plus simple d'allure rectangulaire correspondant au même carré moyen: nous supposerons ainsi que les valeurs probables du champ de fluctuation sont uniformément réparties entre les deux limites $\pm H_{f} \sqrt{ } 3$.

$\S 2$. Les bases de la théorie de l'aimant idéal. Pour obtenir une théorie plus correcte de l'hystérésis, il faut finalement tenir compte:

A) des diverses orientations des grains,

B) de l'effet de proximité,

C) de la dispersion des champs coercitifs des grains individuels,

D) du champ de Lorentz,

E) des fluctuations du champ de Lorentz.

Jusqu'ici, les différentes théories proposées n'ont tenu compte que des points A et B. Nous nous proposons d'esquisser plus loin une théorie schématique de l'aimant permanent idéal qui tienne compte des autres facteurs. Nous supposons que cet aimant est formé d'une phase magnétique (fer par exemple), d'aimantation spontanée $J_{0}=1700$ u.é.m., occupant la fraction $v=0,5$ du volume total dispersée en grains très petits dans une phase non magnétique: l'aimantation à saturation moyenne est donc égale à 850 u.é.m., soit un ordre de grandeur qui convient aussi bien pour les aimants en poudre de fer comprimée que pour les aimants du type Fe-Ni-Al.

Nous supposons que les grains sont assimilables à des ellipsoïdes de révolution allongés possédant toutes les ellipticités possibles de sorte que les champs coercitifs des grains isolés sont dispersés entre 0 , pour la sphère, et $2 \pi J_{0}$, pour le cylindre. Cependant, avec $v=0,5$, l'effet de proximité réduit cette dispersion de moitié, soit entre 0 et $\pi J_{0}$. Pour éviter les grandes complications mathématiques qu'introduit la dispersion des orientations, nous remplaçons les grains de champ critique compris entre $h_{c}$ et $h_{c}+\mathrm{d} h_{c}$, orientés au hasard et correspondant à la fraction $\mathrm{d} J_{s}$ de l'aimantation à saturation, par la distribution suivante beaucoup plus simple:

$\left.1^{\circ}\right)$ des grains d'axe parallèle au champ appliqué, de champ critique $h_{c}^{\prime}=0,54 h_{c}$, correspondant à la fraction $0,50 \mathrm{~d} J_{s}$ de l'aimanta- 
tion à saturation; ces grains fournissent la partie irréversible des variations d'aimantation,

$2^{\circ}$ ) des grains d'axe perpendiculaire au champ appliqué, de même champ critique $h_{c}^{\prime}$ que les précédents et correspondant à la fraction $0,37 \mathrm{~d} J_{s}$ de la saturation; ces grains fournissent la partie réversible des variations d'aimantation.

Ces proportions et ce champ coercitif ont été choisis de manière telle que le cycle d'hystérésis résultant et la courbe de première aimantation (courbe B, fig. 1) de ces deux catégories de grains se rapproche le plus possible des courbes correspondantes relatives à des grains de champ critique $h_{c}$ orientés au hasard (courbe A, fig. 1). D'après ce qui a été dit plus haut, le champ critique supérieur $H_{c}^{\prime}$ est alors égal à $0,54 \pi J_{0}$ soit 2880 u.é.m. Nous admettons dans la suite que les valeurs de $h_{c}^{\prime}$ sont uniformément réparties entre 0 et $H_{c}^{\prime}$.

Nous allons calculer les deux coefficients $a$ et $b$ de Rayleigh et le champ coercitif du modèle ainsi obtenu.

Susceptibilité initiale réversible (terme $a$ de Rayleigh).

Cette susceptibilité initiale provient des grains d'axe perpendiculaire au champ appliqué, soumis en plus de ce dernier à l'action du champ de fluctuation $h_{f}$.

Supposons que la variation totale de l'aimantation macroscopique soit suffisamment faible pour que la topographie du champ de fluctuation puisse être supposée invariable et considérons tous les grains de champ critique compris entre $h_{c}^{\prime}$ et $h_{c}^{\prime}+\mathrm{d} h_{c}^{\prime}$ : ils sont soumis à des champs de fluctuations uniformément répartis entre $-H_{f} \sqrt{ } 3$ et $+H_{f} \sqrt{ } 3$, et, comme nous le montrerons plus loin, $h_{c}^{\prime}$ est toujours inférieur en module à $H_{f} \sqrt{ } 3$. Les grains pour lesquels $h_{f}$ est supérieur à $h_{c}^{\prime}$ sont saturés positivement tandis que les grains pour lesquels $h_{f}$ est inférieur à $-h_{c}^{\prime}$ sont saturés négativement; le moment résultant des grains, pour lesquels $h_{f}$ est compris entre $-h_{c}^{\prime}$ et $+h_{c}^{\prime}$, est nul. Lorsqu'on applique un champ $H$ petit, ces limites $-h_{c}^{\prime}$ et $+h_{c}^{\prime}$ sont respectivement remplacées par $-h_{c}^{\prime}+H$ et $+h_{c}^{\prime}+H$, de sorte que l'application d'un champ $H$ a simplement pour effet de faire passer de $-J_{0}$ à $+J_{0}$ l'aimantation des grains pour lesquels le champ de fluctuation est compris dans un intervalle de largeur égale à $H$ et dont la contribution à l'aimantation à saturation totale est égale à:

$$
\frac{0,37 J_{s} H}{H_{f} \sqrt{ } 3} \frac{\mathrm{d} h_{c}^{\prime}}{H_{c}^{\prime}} .
$$


En ajoutant les contributions des grains correspondant aux différentes valeurs de $h_{c}^{\prime}$, on trouve comme expression de la susceptibilité réversible initiale

$$
a^{\prime}=0,37 J_{s} / H_{f} \sqrt{ } 3
$$

mais nous n'avons pas encore tenu compte du champ de Lorentz qui correspond à un champ magnétisant $N J_{m}$ avec $N=4 \pi / 3$. Comptetenu de ce dernier effet, nous obtenons finalement la valeur définitive $a$ de la susceptibilité réversible initiale

$$
a=\frac{a^{\prime}}{1-N a^{\prime}} .
$$

Il reste à évaluer $H_{f}$, égal à la racine carré du carré moyen du champ de fluctuation pour $J_{m}=0$. Nous utilisons pour cela les formules 5 et 6 ; en admettant que sur les 12 voisins d'un grain donné, il y en a $12 \times 0,50$ dans le sens du champ et $12 \times 0,37$ dans le sens perpendiculaire, on trouve alors

$$
\begin{gathered}
H_{f}=1,932 J_{s}, \\
a^{\prime}=0,11, \quad N a^{\prime}=0,46,
\end{gathered}
$$

et finalement

$$
a=0,20 .
$$

Aimantation irréversible initiale (terme $b$ de Rayleigh).

Elle provient des grains dont l'axe est parallèle au champ appliqué. Un grain de champ critique $h_{c}^{\prime}$, soumis à un champ de fluctuation $h_{f}$, décrit sous l'action d'un champ extérieur un cycle d'hystérésis dyssymétrique rectangulaire, caractérisé par un champ critique inférieur $-h_{c}^{\prime}-h_{f}$ et un champ critique supérieur $h_{c}^{\prime}-h_{f}$. Chacun de ces grains peut être représenté par un point d'un domaine plan, d'abscisse $x=h_{c}^{\prime}-h_{f}$ et d'ordonnée $y=-h_{c}^{\prime}-h_{f}$. Compte-tenu des hypothèses initiales, les points représentatifs des grains du modèle étudié sont uniformément répartis à l'intérieur d'un rectangle ACDE (fig. 2) dont les côtés sont parallèles aux bissectrices des axes ordonnées $\mathrm{OB}$ et $\mathrm{OB}^{\prime}$ et dont les abscisses des sommets $\mathrm{C}$ et $\mathrm{D}$ sont respectivement $H_{c}^{\prime}+H_{f} \sqrt{ } 3$ et $H_{c}^{\prime}-H_{f} \sqrt{ } 3$. Comme $\mathrm{W}$ eis s et Fre u de $\mathrm{nrei} \mathrm{ch}^{6}$ ) l'ont remarqué depuis très longtemps, une telle distribution de cycles asymétriques obéit aux lois de Rayleigh 
relatives aux champs faibles, à savoir

$$
J=a^{\prime} H+b^{\prime} H^{2}
$$

pour la courbe de première aimantation et, pour la courbe de recul décrite à partir du point d'élongation maximum $J_{M}, H_{M}$

$$
J-J_{M}=a^{\prime}\left(H-H_{M}\right)-\frac{b^{\prime}}{2}\left(H-H_{M}\right)^{2} .
$$

Le terme en $a^{\prime}$ correspond naturellement aux grains d'axe perpendiculaire au champ étudiés dans le paragraphe précédent.

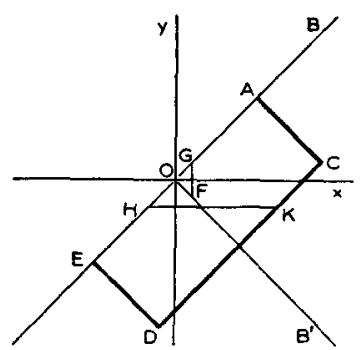

Fig. 2.

Après désaimantation dans un champ alternatif décroissant, les grains dont les points représentatifs sont situés au dessous de $\mathrm{OB}^{\prime}$ sont aimantés positivement: au dessus de $\mathrm{OB}^{\prime}$, ils sont aimantés négativement. Au cours de la première aimantation, l'application du champ $H$ retourne le moment magnétique des grains dont les points représentatifs sont situès dans le triangle $O F G$, avec un côté $F G$ parallèle à $\mathrm{Oy}$ et d'abscisse égale à $H$ (voir à ce sujet: $\mathrm{N}$ é e ${ }^{7}$ ). Il en résulte un terme en $b^{\prime} H^{2}$ avec

$$
b^{\prime}=\frac{0,50 J_{s}}{2 \sqrt{ } 3 H_{c}^{\prime} H_{f}} .
$$

Il faut introduire maintenant le champ de Lorentz que nous avons jusqu'ici négligé, ce qui donne finalement comme valeur du coefficient $b$ du terme irréversible en $H^{2}$ de la loi de première aimantation

$$
b=\frac{b^{\prime}}{\left(1-N a^{\prime}\right)^{3}} .
$$


En introduisant les valeurs numériques adoptées plus haut, on obtient

$$
b^{\prime}=2,6 \cdot 10^{-5} ; \quad b=1,7 \cdot 10^{-4} .
$$

$\mathrm{Ch}$ a m p coercitif. Lorsqu'après avoir aimanté à saturation, on applique un champ magnétique négatif le champ coercitif - $H_{c}$ est atteint lorsque l'aimantation résultante est nulle. En ce qui concerne les grains dont l'axe est parallèle au champ, on montre facilement ${ }^{7}$ ) qu'en traçant sur la figure 2 une droite $\mathrm{HK}$ parallèle à $\mathrm{Ox}$, d'ordonnée $-H_{c}$, les grains du domaine trapézoïdal HKCA sont aimantés négativement tandis que ceux du domaine HEDK sont aimantés positivement. L'aimantation résultante correspondante est égale à

$$
\frac{0,50 J_{s}}{2 \sqrt{3} H_{c}^{\prime} H_{f}}\left[H_{c}^{2}-\left(H_{c}^{\prime}-H_{c}\right)^{2}\right] .
$$

D'autre part, l'aimantation réversible des grains d'axe perpendiculaire au champ est égale à

$$
\frac{0,37 J_{s} H_{c}}{H_{f} \sqrt{ } 3}
$$

et en écrivant que l'aimantation totale, égale à la somme des deux termes précédents, est nulle, on obtient la relation

$$
H_{c}=0,287 H_{c}^{\prime} \text {. }
$$

Avec les valeurs numériques déjà adoptées, il vient

$$
H_{c}=830 .
$$

§3. Comparaison avec les résultats expérimentaux. Comparons maintenant les valeurs ainsi obtenues aux données expérimentales typiques relatives aux aimants en poudre de fer comprimée ou aux aimants fer-nickel-aluminium. Nous obtenons le tableau suivant:

\begin{tabular}{|l|c|c|c|}
\hline & $a$ & $b$ & $H_{c}$ \\
\hline théorie & 0,20 & $1,7 \cdot 10^{-4}$ & 830 \\
fer divisé & 0,25 & $2,7 \cdot 10^{-4}$ & 473 \\
Fe-Ni-Al & 0,26 & $1,8 \cdot 10^{-4}$ & 557 \\
\hline
\end{tabular}

L'aimant idéal de notre théorie possède ainsi des caractéristiques 
étonnament voisines de celles des aimants réels, compte-tenu des très grossières approximations qui ont été faites plus haut.

Il n'y a pas à s'étonner par exemple que la théorie fournisse un champ coercitif plus grand que ceux qui ont jamais été obtenus pour ces types d'aimants: on obtiendrait en effet 550 à la place de 830 si on supposait que les formes possibles des grains, au lieu d'être réparties entre la sphère et le cylindre, étaient réparties entre la sphère et des ellipsoïdes d'allongement 3 , ce qui est certainement beaucoup plus conforme à la réalité.

Il y aurait lieu maintenant de compléter l'étude du modèle dans le domaine des grandes aimantations et de calculer par exemple l'aimantation rémanente et la susceptibilité anhystérétique: ce serait assez compliqué car il faudrait tenir compte du fait que le carré moyen du champ de fluctuation est proportionel à $J_{s}^{2}-J_{m}^{2}$ et, puisqu'il s'agit de grandes amplitudes de variation d'aimantation, faire intervenir la répartition des champs de fluctuation suivant une loi de Gauss. Des calculs provisoires assez pénibles paraissent donner un ordre de grandeur correct. Remarquons en passant qu'avec cette nouvelle manière de voir il n'y a plus aucune raison d'obtenir une aimantation rémanente exactement égale à la moitié de l'aimantation à saturation.

$\S 4$. Conclusions. Ainsi, en introduisant et en mettant en oeuvre d'une manière très simple les interactions entre les domaines sous la forme d'une sorte de champ de Lorentz et de ses fluctuations, on obtient un modèle qui possède des propriétés magnétiques très voisines de celles des substances dures.

On peut notamment interpréter quantitativement la valeur de la susceptibilité réversible et expliquer pourquoi ces substances dures obéissent aux lois de Rayleigh aussi bien que les substances douces pour lesquelles l'interprétation, entièrement différente, est basée sur le déplacement des parois. Il est tout-à-fait intéressant de remarquer que le mécanisme que nous avons étudié ici fournit une valeur de $b H_{c} / a$ égale à 0,70 qui est d'un ordre de grandeur très voisin de la valeur 0,42 fournie par la théorie des lois de Rayleigh pour les déplacements des parois quand celles-ci sont très rapprochées les unes des autres ${ }^{7}$ ). Nous avions déjà fait remarquer autrefois ${ }^{8}{ }^{9}$ ) qu'en ce qui concerne le champ coercitif la théorie des déplacements de paroi et la théorie des rotations fournissaient dans de telles conditions des résultats 
convergents. Il en est de même ici. Cette constatation est très réconfortante car ces deux théories se rapportent à deux manières entièrement différentes d'aborder le problème des substances magnétiques denses très perturbées: dans l'une on part d'un grand cristal peu perturbé, dans l'autre on part de grains très fins isolés les uns des autres. On aboutit cependant à des résultats voisins.

Manuscrit reçu le 7-9-53.

\section{BIBLIOGRAPHIE}

1) A k u lov, N., Z. Phys. 81 (1933) 790.

2) Né e 1, L., Compt. Rend. a24 (1947) 1488, 1550.

3) St on er, E. C. and E. P. Wo h lf a r th, Phi. Trans. 240 (1948) 599.

4) N é e 1, L., Ann. Géophys. 5 (1949) 99.

5) W e i 1, L., Compt. Rend. 225 (1947) 229; 297 (1948) 1347.

6) Weiss, P. and J. P. de Freudenrei c h, Arch. Sci. Natur. Genève, 19 (19) 449.

7) Née 1, L., Cahiers de Physique 1942, no. 12, p. 1; 1943, no. 13, p. 18.

8) N é e 1, L., Ann. Univ. Grenoble 22 (1946) 299.

9) N é e 1, L., Physica 15 (1949) 225. 